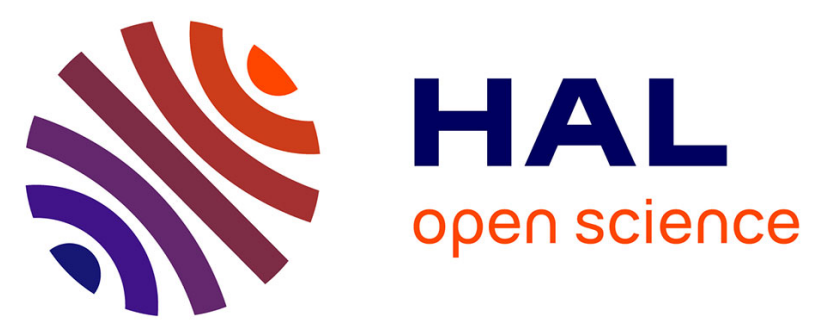

\title{
On the mechanics of magnetic fluids with field-induced phase transition: application to Couette flow
}

\author{
V G Bashtovoi, P. Kuzhir, A. y Zubarev, V S Moroz
}

\section{To cite this version:}

V G Bashtovoi, P. Kuzhir, A. y Zubarev, V S Moroz. On the mechanics of magnetic fluids with fieldinduced phase transition: application to Couette flow. Magnetohydrodynamics c/c of Magnitnaia Gidrodinamika, 2018, 54 (3), pp.181-196. hal-01970785

\section{HAL Id: hal-01970785 \\ https://hal.science/hal-01970785}

Submitted on 16 Jan 2019

HAL is a multi-disciplinary open access archive for the deposit and dissemination of scientific research documents, whether they are published or not. The documents may come from teaching and research institutions in France or abroad, or from public or private research centers.
L'archive ouverte pluridisciplinaire HAL, est destinée au dépôt et à la diffusion de documents scientifiques de niveau recherche, publiés ou non, émanant des établissements d'enseignement et de recherche français ou étrangers, des laboratoires publics ou privés. 
On the mechanics of magnetic fluids with field-induced phase transition: application to Couette flow

\author{
V.G. Bashtovoi ${ }^{\text {a, }}$, P.P. Kuzhir ${ }^{b}$, A.Y. Zubarev ${ }^{c}$, V.S. Moroz ${ }^{\mathrm{a}}$ \\ a Belarusian National Technical University, Laboratory of Thermomechas of Magnetic Fluids, 65, \\ Nezavisimosti Ave., Minsk, 220013, Belarus \\ ${ }^{\mathrm{b}}$ University Côte d'Azur, CNRS UMR 7010, Institute of Physics of Nice, Parc Valrose, Nice \\ 06108, France \\ ${ }^{\mathrm{c}}$ Ural Federal University, Department of Theoretical and Mathematical Physics, 51, Lenina Ave., \\ 620083, Ekaterinburg, Russian Federation \\ *Corresponding author: Bashtovoi V.G., Tel.:+375-29-3261723, Fax:+375-17-3310052. \\ E-mail address: bashv@rambler.ru (V.G. Bashtovoi)
}

Key words: magnetic fluid; magnetic field; magnetophoresis; brownian diffusion; phase transition; Couette flow.

\begin{abstract}
Influence of processes of Brownian diffusion and magnetophoresis which are followed by phase transition on characteristics of a stationary plane Couette flow of a magnetic fluid in non uniform magnetic field is considered. Conditions of phase transition in magnetic fluid are accepted as a natural restriction of increasing of concentration of particles in non-uniform magnetic field. Profiles of concentration of particles are calculated and dependences of volume magnetic force, and also viscous force from them are established.
\end{abstract}

\title{
1. Introduction
}

In this paper magnetic fluid is a stable suspension of magnetic nanoparticles with size of order $10 \mathrm{~nm}$ and classical magnetic and mechanical properties [1-4]. Stability of suspension is provided by the special surfactant.

The nanosize of particles provides a Brownian nature of their behavior in fluid, exchange of a momentum with molecules of fluid, transfer of a momentum resulted by their interaction with external magnetic field to all volume of fluid. Due to this such suspension can be considered as a continuous medium with magnetization providing the volume magnetic force in fluid in external magnetic field.

In non-uniform magnetic field the natural processes taking place in such system are magnetophoresis and Brownian diffusion of magnetic particles leading to a redistribution of concentration of particles. On the importance of these processes in magnetic fluids the attention was paid in the very first works on this subject and in the subsequent monographs [1-3].

These processes become apparent significantly in magnetic fluid seals due to large gradients of magnetic field intensity using in them [4-6]. 
Recently it is specified also an important role which these processes can play in the general problems of statics and dynamics of magnetic fluids due to their influence on distribution of magnetization, volume magnetic force, and viscous tension in fluids. It is important, for example, at a levitation of magnetic and non magnetic bodies in them as applied to magnetic fluid suspensions, supports, vibroprotective systems [6 - 8], and also at a flow of magnetic fluids in channels [9]. In these works the complete system of the differential equations and boundary conditions providing the adequate description of these processes is formulated.

The main disadvantage of the presented description of processes of Brownian diffusion and magnetophoresis in magnetic fluids based on the classical theory of these processes is that they give unlimited increasing of concentration of magnetic particles in areas with high intensity of non-uniform magnetic field, more than at densest packing. It results, in particular, abnormally high values of their viscosity and, respectively, viscous tension at their flow [9]. From the physical point of view it cannot be accepted.

However, it is known that even in a non magnetic suspension the increasing of concentration of particles leads to a phase transition [10]. It means a sharp transition of suspension to the high-concentrated state of particles in which proposed description of diffusive processes is not valid but which has fluidity and a finite viscosity.

The situation is equivalent to that which arises upon usual phase transition of the gaseous medium to a liquid phase at its condensation.

It is obvious that the corresponding critical values of concentration of particles leading to such phase transition have to be a natural limit of validity of the theory of Brownian diffusion for suspension.

It is known also that in magnetic fluid as a suspension of magnetic particles, the special phase transition to the high-concentrated state of particles takes place [2, 11-17].

It is caused by the interaction of magnetic particles with each other, and with external magnetic field. In spite of the fact that properties of such high-concentrated magnetic fluid still are studied insufficiently, there is rather large number of the experimental data demonstrating it behavior as a liquid medium. First of all it belongs to data on deformation of drops of this phase in magnetic fields [2] and a possibility to characterize them by the corresponding surface tension and viscosity.

\section{General theory.}

We believe that magnetic particles in magnetic fluid at steady state can be in two thermodynamic states: 1) disordered weak-concentrated phase with behavior of magnetic particles in fluid, similar to gaseous state (a "gas" phase), and 2) a highconcentrated phase with ordered structured packing of magnetic particles and their behavior, similar to a condensed incompressible fluid (the "condensed" phase).

Further the parameters relating to magnetic fluid with "gaseous" and the "condensed" phases of magnetic particles will be noted by indexes $g$ and $c$ respectively.

The main properties of these phases are accepted by the following.

2.1. Mass transfer processes.

a) "Gas" phase. 
According to the approach developed in [6-9] on the base of phenomenological theory of mass transfer the distribution of volume concentration $\Phi_{g}$ of magnetic particles having density $\rho$ in a "gas" phase is governed by the diffusion equation

$$
\rho \frac{\partial \Phi_{g}}{\partial t}+\operatorname{div} \vec{i}=0
$$

where mass flux density of particles $\vec{i}$ in motionless fluid at the presence of nonuniform magnetic field with value of intensity $H$ is defined as follows

$$
\vec{i}=-\rho D \nabla \Phi_{g}+\rho b \mu_{0} m \Phi_{g} L(\xi) \nabla H,
$$

where: $t$ is time, $D$ - diffusion coefficient, which is connected with the mobility of particles $b$ by the Einstein's relation $D=k T b ; \mu_{0} \approx 1,26 \cdot 10^{-6} \mathrm{H} / \mathrm{m}$ - magnetic permeability of vacuum; $m$ - magnetic moment of individual particle, J/T; $k \approx 1,38 \cdot 10^{-23}$ $\mathrm{J} / \mathrm{K}$ - Boltzmann constant; $T$ - temperature, $\mathrm{K} ; L(\xi)=c t h(\xi)-1 / \xi$ - Langevin function, $\xi=\mu_{0} m H / k T$. In general case diffusion coefficient $\mathrm{D}$ is a function of concentration $\Phi_{g}$.

On boundaries impermeable for particles the normal component of mass flux density $i_{n}$ must be equal to zero.

In accordance with (1) at stationarity mass flux density $\vec{i}$ is equal to zero in whole volume of fluid bounded of impermeable boundaries. Taking into account (2) and Einstein's relation it means

$\nabla \Phi_{g}=\left(\mu_{0} m / k T\right) \Phi_{g} L(\xi) \nabla H$

The dimension analysis of the equations (3) shows that a stationary problem described by them is defined by one dimensionless parameter $U=\mu_{0} m H_{*} / k T$ representing the ratio of magnetic energy of a particle to its thermal energy [6-9]. It includes characteristic value of magnetic field intensity $H_{*}$ specified for each concrete situation.

Most often typical magnetic fluid contains as a magnetic phase particles of magnetite with magnetization of $4,46 \cdot 10^{5} \mathrm{~A} / \mathrm{m}$ [1]. At their characteristic diameters $d$ about $10^{-8} \mathrm{~m}$ and, respectively, a volume of $5,2 \cdot 10^{-25} \mathrm{~m}^{3}$, the magnetic moment of particle $m$ is about $2,3 \cdot 10^{-19} \mathrm{~J} / \mathrm{T}$. Then, at room temperature $T=300 \mathrm{~K}$ value $U$ of an order of one takes place at characteristic magnetic field intensity $H_{*}$ of about $10^{4} \mathrm{~A} / \mathrm{m}$. In real situations, for example, in gaps of magnetic fluid seal in which strong magnetic field to $10^{6} \mathrm{~A} / \mathrm{m}$ with large gradients the values of parameter $U$ can reach several tens.

Total volume of magnetic particles $V_{m g}$ in the volume $V_{g}$, occupied by this phase is equal $V_{m g}=\int_{V g} \Phi_{g} d V$.

b) “Condensed” phase. 
Analysis shows that in the condensed phase the volume fraction of nanoparticles varies relatively insignificantly, and remains quite close to the maximum packing fraction of the considered particle network. At such condition we can assume that the "condensed" phase has constant concentration of magnetic particles, $\Phi_{\mathrm{c}}=$ Const corresponding to conditions of thermodynamic coexistence of phases.

The total volume of magnetic particles $V_{m c}$ in volume $V_{c}$ occupied by this phase, is equal to $V_{m c}=\int_{V c} \Phi_{c} d V=\Phi_{c} V_{c}$.

Magnetic interaction of spherical ferromagnetic magnetic particles has a dipole dipole character. It is defined by the magnetic moment of a particle $m$, and characterized by dimensionless parameter $\lambda=\left(\mu_{0} m^{2}\right) /\left(4 \pi d^{3} k T\right)$ representing the ratio of the energy of dipole - dipole interaction of two closely situated particles to the thermal energy.

For magnetite particles with the characteristics given above and at the room temperature parameter $\lambda$ is about 1,3 .

c) An average concentration of particles $\Phi_{0}$ in all considered volume $V$ of magnetic fluid is a constant and is determined by the next way

$\frac{1}{V}\left[\int_{V g} \Phi_{g} d V+\int_{V c} \Phi_{c} d V\right]=\frac{1}{V}\left[\int_{V g} \Phi_{g} d V+\Phi_{c} V_{c}\right]=\Phi_{0}$.

\subsection{Phase co-existence.}

Interface between both phases and concentration of particles in the "condensed" phase are defined by thermodynamic conditions of coexistence of phases. The approach developed in [11-14] is adopted in the present paper. It leads to convenient analytical relationships of phase coexistence in the magnetic fluid taking into account dipolar interaction between magnetic particles. This approach was effectively used for describing of experimental results in [14]. The phase equilibrium between two phases is given by the equilibrium of their chemical potentials and osmotic pressures.

The chemical potential $\zeta(\Phi, \xi, \lambda)$ and the osmotic pressure $p(\Phi, \xi, \lambda)$, both contain the contributions coming from and hard-sphere repulsion $\zeta_{h s,} p_{h s}$ and magnetic interactions $\zeta_{m}, p_{m}$.

The appropriate expressions for the first kind of interaction have been developed on the basis of osmotic compressibility calculations carried out by Carnahan and Starling [18] for semidilute and Hall [19] for concentrated hard-sphere suspensions.

Based on the perturbation analysis, Buyevich and Ivanov [20] have derived the expressions for the magnetic contribution to the chemical potential and osmotic pressure of magnetic particles in a magnetic fluid taking into account pair magnetic interactions between particles and neglecting higher order interactions.

Assuming that the steric interactions between particles in magnetic fluid respect the Carnahan-Starling law in the "gas" phase and the Hall law in the "condensed" phase, by using the results [20], we arrive to the following expressions for quantities $\zeta$ and $p$ in both phases. 


$$
\begin{aligned}
& \zeta_{g}\left(\Phi_{g}, \xi, \lambda\right)=\left(\zeta_{h s}\right)_{g}+\left(\zeta_{m}\right)_{g}=k T\left[\ln \Phi_{g}+\Phi_{g} \frac{8-9 \Phi_{g}+3 \Phi_{g}^{2}}{\left(1-\Phi_{g}\right)^{3}}+\ln \frac{\xi}{\operatorname{sh} \xi}-\right. \\
& \left.-8\left(L^{2}(\xi) \lambda+\frac{1}{3} \lambda^{2}\right) \Phi_{g}\right], \\
& p_{g}\left(\Phi_{g}, \xi, \lambda\right)=\left(p_{h s}\right)_{g}+\left(p_{m}\right)_{g}=\frac{k T}{V_{p}}\left[\Phi_{g} \frac{1+\Phi_{g}+\Phi_{g}^{2}-\Phi_{g}^{3}}{\left(1-\Phi_{g}\right)^{3}}-\right. \\
& \left.-4\left(L^{2}(\xi) \lambda+\frac{1}{3} \lambda^{2}\right) \Phi_{g}^{2}\right], \\
& \zeta_{c}\left(\Phi_{c}, \xi, \lambda\right)=\left(\zeta_{h s}\right)_{c}+\left(\zeta_{m}\right)_{c}=k T\left[\frac{A}{\Phi_{m}} \ln \frac{\Phi_{c}}{\Phi_{m}-\Phi_{c}}+\frac{A}{\Phi_{m}-\Phi_{c}}+C+\ln \frac{\xi}{\operatorname{sh} \xi}-\right. \\
& \left.-8\left(L^{2}(\xi) \lambda+\frac{1}{3} \lambda^{2}\right)_{\Phi_{c}}\right], \\
& p_{c}\left(\Phi_{c}, \xi, \lambda\right)=\left(p_{h s}\right)_{c}+\left(p_{m}\right)_{c}=\frac{k T}{V_{p}}\left[\Phi_{c} \frac{A}{\Phi_{m}-\Phi_{c}}-4\left(L^{2}(\xi) \lambda+\frac{1}{3} \lambda^{2}\right) \Phi_{c}^{2}\right]
\end{aligned}
$$

Here $V_{p}$ is volume of the particle, $\Phi_{\mathrm{m}}$ is the volume concentration of densest packed particles (for the face-centered cubic (FCC) lattice $\Phi_{m}=0.74$ ), A and C are some numerical parameters, estimated in [10] as $A=2.2, C=1.255$.

In reality, at high enough field, a body-centered tetragonal (BCT) structure exists instead of the FCC lattice, however, the energy and magnetic properties of both lattices are quite close, so that the assumption of the FCC lattice should not generate significant errors in calculations of the concentration profiles. The assumption of the FCC lattice allows us to recover the order-disorder phase transition in the absence of the magnetic field.

Phase co-existence is defined by the equality of their chemical potentials and osmotic pressure on the phase interface:

$$
\zeta_{g}\left(\Phi_{g}, \xi, \lambda\right)=\zeta_{c}\left(\Phi_{c}, \xi, \lambda\right), p_{g}\left(\Phi_{g}, \xi, \lambda\right)=p_{c}\left(\Phi_{c}, \xi, \lambda\right) .
$$

Taking into account (5)-(8) it leads to the next equations for determination of concentrations on the interface: 


$$
\begin{aligned}
& \ln \Phi_{g}+\Phi_{g} \frac{8-9 \Phi_{g}+3 \Phi_{g}^{2}}{\left(1-\Phi_{g}\right)^{3}}-8\left[L^{2}(\xi) \lambda+\frac{1}{3} \lambda^{2}\right] \Phi_{g}= \\
& =\frac{A}{\Phi_{m}} \ln \frac{\Phi_{c}}{\Phi_{m}-\Phi_{c}}+\frac{A}{\Phi_{m}-\Phi_{c}}+C-8\left[L^{2}(\xi) \lambda+\frac{1}{3} \lambda^{2}\right] \Phi_{c}, \\
& \Phi_{g} \frac{1+\Phi_{g}+\Phi_{g}^{2}-\Phi_{g}^{3}}{\left(1-\Phi_{g}\right)^{3}}-4\left[L^{2}(\xi) \lambda+\frac{1}{3} \lambda^{2}\right] \Phi_{g}^{2}= \\
& =\Phi_{c} \frac{A}{\Phi_{m}-\Phi_{c}}-4\left[L^{2}(\xi) \lambda+\frac{1}{3} \lambda^{2}\right] \Phi_{c} .
\end{aligned}
$$

The equations (10), (11) have to be solved numerically with respect to the concentrations $\Phi_{\mathrm{g}}$ and $\Phi_{\mathrm{c}}$ for arbitrary values of magnetic field intensity $\xi$ and parameter $\lambda$. As a result the diagrams of phase equilibrium $\Phi_{\mathrm{g}}(\xi, \lambda)$ and $\Phi_{\mathrm{c}}(\xi, \lambda)$, defining interface between the "gas" and "condensed" phases in magnetic fluid can be found and constructed.

So, for example, in the absence of magnetic field $(\xi=0)$ and for nonmagnetic particles $(\lambda=0)$ these equations have a solution: $\Phi_{\mathrm{g}}=0,495$ and $\Phi_{\mathrm{c}}=0,545$ [10].

In the case of magnetic fluid magnetized to saturation, when $\xi>>1$ and $L(\xi) \approx 1$, diagrams of phase equilibrium, calculated on the base of equations (10)-(11), are presented in Fig. 1.

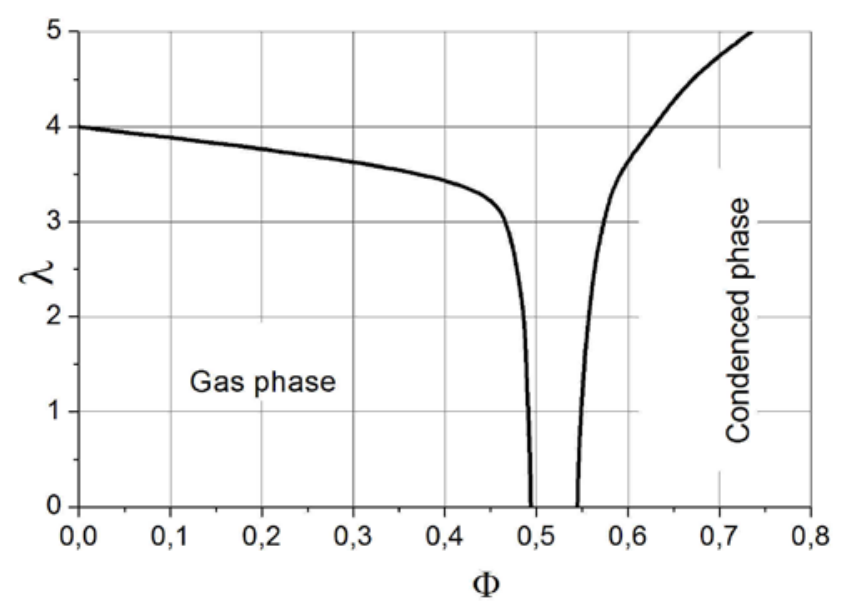

Fig. 1. Diagram of phase equilibrium in magnetic fluid with magnetization of saturation.

The both binodal curves divide the space of diagram into the two regions: 1) the disordered fluid - "gas" phase as a continuum under the left curve, and 2) the "condensed" phase as a continuum under the right curve. At given value of $\lambda$ the points on the left curve define a value of critical concentration of particles in "gas" phase $\Phi_{\mathrm{g}}$ at which phase transition arises. And the corresponding points on the right curve determine a value of concentration of particles in "condensed" phase $\Phi_{c}$. 
In stationary equilibrium "gas" and "condensed" phases as a continuum coexist with interface between them. There is a jump of concentration between phases on this interface which is determined by their corresponding values on binodal curves.

Fig.1 demonstrates that significant discrepancy between binodal curves is appeared at $\lambda \geq 3$. That is, taking into account the interaction between particles $(\lambda \neq 0)$ in the equations (10), (11) does jump of concentration of particles between the "gas" and "condensed" phases to much more considerable, than 0,495 and 0,545 which is characteristic of suspensions of non magnetic particles [10]. More precise critical values of $\Phi_{\mathrm{g}}$ and $\Phi_{\mathrm{c}}$ for some values of parameter $\lambda$ corresponding to the Fig.1 are presented in the table below.

Table 1. Critical concentration of particles in "gas" phase $\Phi_{\mathrm{g}}$ and corresponding concentration $\Phi_{c}$ of particles in "condensed" phase at different values of $\lambda$ at phase transition in magnetic fluid at magnetic saturation.

\begin{tabular}{|l|l|l|l|l|l|l|l|l|l|l|l|}
\hline$\lambda$ & 0 & 0,5 & 1 & 1,5 & 2 & 2,5 & 3 & 3,5 & 4 & 4,5 & 5 \\
\hline$\Phi_{\mathrm{g}}$ & 0,494 & 0,493 & 0,491 & 0,489 & 0,487 & 0,479 & 0,469 & 0,440 & $3,69 \cdot 10^{-8}$ & $2,37 \cdot 10^{-10}$ & $8,69 \cdot 10^{-13}$ \\
\hline$\Phi_{\mathrm{c}}$ & 0,545 & 0,546 & 0,549 & 0,552 & 0,557 & 0,563 & 0,573 & 0,587 & 0,649 & 0,666 & 0,735 \\
\hline
\end{tabular}

The analysis of the equations (10), (11) shows that at large values of parameter $\lambda$ critical value of concentration of particles in a "gas" phase $\Phi_{\mathrm{g}}$ asymptotically tends to zero, and concentration of particles in the "condensed" phase $\Phi_{\mathrm{c}}$ asymptotically tends to the maximum value $\Phi_{\mathrm{m}}$. Equating the right part of eq.(11) to zero, in the case of strong magnetic field $(L(\xi) \approx 1)$ one can get $\left(\Phi_{m}-\Phi_{c}\right) \Phi_{c}=A / 4\left(\lambda+\lambda^{2} / 3\right)$.

Similarly the equation (10) taking into account (11) in this case gives asymptotic dependence $\Phi_{g}=\exp \left[-4\left(\lambda+\lambda^{2} / 3\right) \Phi_{c}\right]$.

We suppose also that:

2.3. Magnetization of magnetic fluid $M$ at any phase state of magnetic particles depends on their concentration $\Phi$, magnetization of individual magnetic particle $M_{m}$ and on intensity of magnetic field under Langevin function: $M=M_{m} \Phi L(\xi)$.

As shown in [20], the dipole-dipole interaction between the particles leads to modification of the Langevin's law of magnetization of the fluid and the form $M=M_{m} \Phi(x) L(\xi)\left[1+8 \lambda \Phi(x) \frac{d L(\xi)}{d \xi}\right]$ can be used.

Magnetization of magnetic particles $M_{m}$ is supposed uniform in their volume and is equal to the ratio of their magnetic moment $m$ to their volume $V_{p}: M_{m}=m / V_{p}$.

2.4. Magnetic fluid at any phase state of magnetic particles is Newtonian and is governed by the well known equations of mechanics of magnetic fluids [1-3].

2.5. The magnetic force $\vec{F}_{m}$ acting on the volume $V$ of magnetic fluid in nonuniform magnetic field is determined by expression:

$$
\vec{F}_{m}=\mu_{0} \int_{V} M \nabla H d V=\mu_{0} M_{m} \int_{V} \Phi L(\xi) \nabla H d V .
$$


2.6. A dynamic coefficient of magnetic fluid viscosity $\eta$ at any phase of magnetic particles will be described by the approximation of Krieger-Dougherty [21, 22]

$\eta=\eta(\Phi=0)\left(1-\frac{\Phi}{\Phi_{m}}\right)^{-2,5 \Phi m}$,

where $\eta(\Phi=0)$ is a dynamic viscosity of a base fluid, and $\Phi_{\mathrm{m}}$ is a concentration at densest packing of particles. At FCC lattice $\Phi_{\mathrm{m}}=0,74$ and

$\eta=\eta(\Phi=0)(1-1,35 \Phi)^{-1,85}$

\section{Couette flow of magnetic fluid with phase transition.}

We will consider influence of mass transfer processes, leading to the phase transition in magnetic fluid, on the stationary plane Couette flow in locally non uniform magnetic field as is presented in Fig. 2.

It will allow us to describe problem by exact analytical solutions of equation (3) and find out all of the main features of this influence. Also this problem can be a good model for description of a magnetic fluid behavior in a gap of magnetic fluid seal $[6,9]$.

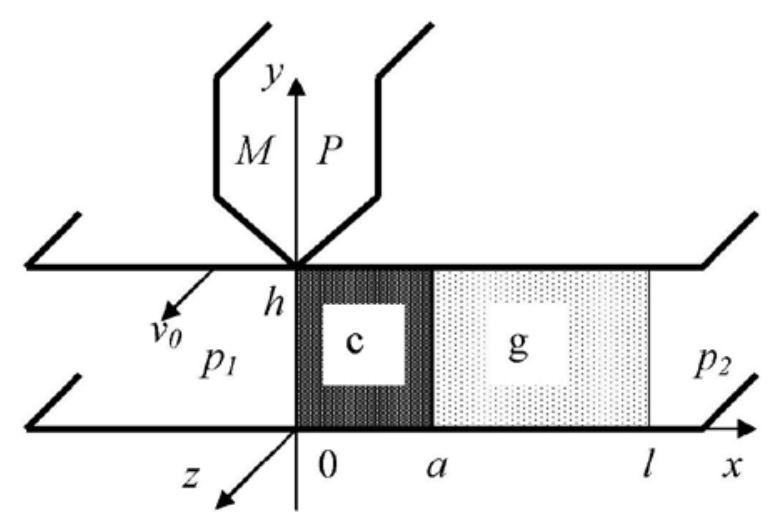

Fig. 2. Geometry of the problem.

$c$ - region of condenced phase, $g$ - region of the gas phase, MP - pole of the magnet system.

Let, as shown in Fig.2, the volume of magnetic fluid $(0 \leq x \leq l, 0 \leq y \leq h)$ fill a gap between two plane-parallel solid boundaries $y=0$ and $y=h$. Side boundaries $x=0$ and $x=l$ of the fluid volume are supposed plane too. It means that the curvature of these boundaries caused by the wetting of solid boundaries is not essential for the being studied processes. Magnetic fluid is held in the gap by the non-uniform magnetic field $H(x)$ created by the local pole of a magnet system (MP) when pressure drop along the $\mathrm{x}$ axis $\Delta p=p_{1}-p_{2}$ is applied to it. This position of magnetic fluid volume corresponds to the maximum pressure drop which is held by magnetic fluid seal. If magnetic field depends only on coordinate $x$, the distribution of concentration of particles in fluid will be function only of coordinate $x$ too. 
The part of the fluid located in $0 \leq x \leq a$, i.e. near a pole in the area with maximum intensity of magnetic field, can represent the "condensed" phase of magnetic particles, and the area $a \leq x \leq l-$ the "gas" phase. Location of the interface between these phases $x=a$ is to be determined in the process of the solution of equations (10)(11).

We suppose that the upper boundary $(y=h)$ moves relatively to the bottom one $(y=0)$ with the velocity $v_{0}$ in the direction of $z$ axis. The Couette flow with velocity profile $v_{z}=v_{0} y / h$ takes place in the fluid.

Viscous tension $\tau$ on the upper boundary is equal: $\tau=\eta\left(\partial v_{z} / \partial y\right)=\eta v_{0} / h$ and is determined by the dynamic coefficient of viscosity of the fluid $\eta[\Phi(\mathrm{x})]$ which is a function of particle concentration in accordance to (13).

Further consideration will be implemented by using the dimensionless parameters: $x^{\prime}=x / l, H^{\prime}=H / H_{*}$, where $H_{*}$ is a maximum value of magnetic field intensity at $x=0: H_{*}=H(0)$, and the dimensionless coordinate $s$ of interface between phases is $s=a / l$.

All boundaries of the magnetic fluid volume will be assumed impermeable for particles and, as it was mentioned above, the mass flux density in whole volume of the fluid is zero. In accordance with the equation (3), it means that distribution of particles concentration in the "gas" phase will be governed by the next dimensionless equation

$$
\frac{d \Phi_{g}}{d x^{\prime}}=U \Phi_{g} L\left(U H^{\prime}\right) \frac{d H^{\prime}}{d x^{\prime}}, \quad U=\mu_{0} m H_{*} / k T,
$$

which has the general solution

$$
\Phi_{g}=B \frac{\operatorname{sh}\left[U H^{\prime}\left(x^{\prime}\right)\right]}{H^{\prime}\left(x^{\prime}\right)} .
$$

At the absence of phase transition constant $B$ is defined from the condition (4) as

$$
\int_{0}^{1} \Phi_{g} d x^{\prime}=\Phi_{0} \text { and } B=\Phi_{0}\left\{\int_{0}^{1} \frac{s h\left[U H^{\prime}\left(x^{\prime}\right)\right]}{H^{\prime}\left(x^{\prime}\right)} d x^{\prime}\right\}^{-1} \text {. }
$$

At the presence of the phase transition we will use the next approach.

Let the critical value of particle concentration in the "gas" phase on the interface with "condensed" phase $x=s$ be $\Phi_{\mathrm{g}}(s)=\Phi_{\mathrm{g}^{*}}$. Than the constant $B$ in (15) is defined as

$$
B=\Phi_{g^{*}} \frac{H^{\prime}(s)}{\operatorname{sh}\left[U H^{\prime}(s)\right]} .
$$


In this case the coordinate $s$ of the interface between the phases is determined from the condition of constancy of average concentration of particles (4)

$$
\begin{aligned}
& \Phi_{c} s+\int_{s}^{1} \Phi_{g} d x^{\prime}=\Phi_{0} \text { as } \\
& \Phi_{c} s+\Phi_{g^{*}} \frac{H^{\prime}(s)}{s h\left[U H^{\prime}(s)\right]} \int_{s}^{1} \frac{s h\left(U H^{\prime}\right)}{H^{\prime}} d x^{\prime}=\Phi_{0} .
\end{aligned}
$$

This equation must be solved for $s$ at predetermined configuration of magnetic field $H\left(x^{\prime}\right)$, values of parameters $U$ and $\Phi_{0}$, with taking into account equations (10), (11) for $\Phi_{\mathrm{c}}$ and $\Phi_{\mathrm{g} *}$.

In the case when overwhelming majority of particles at large values of magnetic field intensity is concentrated in the "condensed" phase near the pole of magnet in the region $0 \leq x^{\prime} \leq s$, the first term in the left part of (18) becomes much more then second one and this equation gives a linear dependence of $s$ on $\Phi_{0}$ :

$s=\Phi_{0} / \Phi_{c}$.

Influence of redistribution of particles concentration on viscous and magnetic forces acting in the considered volume of magnetic fluid is of considerable interest for this problem.

If $w$ is a length of the layer in the direction of axis $z$ viscous force $F_{\tau}$ acting on the moving upper boundary of the layer is

$$
F_{\tau}=w \int_{0}^{l} \tau(y=h) d x=\left(w v_{0} / h\right) \int_{0}^{l} \eta[\Phi(x)] d x .
$$

Value of this force $F_{\tau 0}$ for the initial homogeneous fluid with constant concentration of particles $\Phi_{0}$ and constant dynamic viscosity $\eta_{0}=\eta\left(\Phi_{0}\right)$, will be equal to $F_{\tau 0}=\left(w v_{0} l \eta_{0} / h\right)$.

It is convenient to describe the influence of concentration redistribution on this force by using the dimensionless coefficient $k_{\tau}=F_{\tau} / F_{\tau 0}=\int_{0}^{1} \eta^{\prime}\left[\Phi\left(x^{\prime}\right)\right] d x^{\prime}$, where $\eta^{\prime}=\eta / \eta_{0}$

At the absence of phase transition we get $k_{\tau}=\int_{0}^{1} \eta^{\prime}\left[\Phi_{g}\left(x^{\prime}\right)\right] d x^{\prime}$, whereas at the presence of the phase transition

$$
k_{\tau}=\int_{0}^{s} \eta^{\prime}\left(\Phi_{c}\right) d x^{\prime}+\int_{s}^{1} \eta^{\prime}\left[\Phi_{g}\left(x^{\prime}\right)\right] d x^{\prime}=\eta^{\prime}\left(\Phi_{c}\right) s+\int_{s}^{1} \eta^{\prime}\left[\Phi_{g}\left(x^{\prime}\right)\right] d x^{\prime} .
$$

Value of magnetic force $F_{m}$ acting on the magnetic fluid volume along $x$ axis will be defined from (12) as: 
$F_{m}=\mu_{0} M_{m} w h \int_{1}^{0} \Phi(x) L(\xi) \frac{d H}{d x} d x$

Value of dimensionless magnetic force $f_{m}=F_{m} /\left(w h \mu_{0} M_{m} H_{*}\right)$ will be defined by the next way:

a) at the absence of phase transition

$f_{m}=\int_{1}^{0} L\left[U H^{\prime}\left(x^{\prime}\right)\right] \Phi_{g} \frac{d H^{\prime}}{d x^{\prime}} d x^{\prime}$, or with taking into account (14),

$f_{m}=\frac{1}{U} \int_{1}^{0} \frac{d \Phi_{g}}{d x^{\prime}} d x=\frac{1}{U}\left[\Phi_{g}(0)-\Phi_{g}(1)\right]$

b) at the presence of phase transition

$$
\begin{aligned}
& f_{m}=\int_{1}^{s} L\left[U H^{\prime}\left(x^{\prime}\right)\right] \Phi_{g} \frac{d H^{\prime}}{d x^{\prime}} d x^{\prime}+\Phi_{c} \int_{H^{\prime}(s)}^{H^{\prime}(0)} L\left(U H^{\prime}\right) d H^{\prime}= \\
& =\frac{1}{U}\left[\Phi_{g}(s)-\Phi_{g}(1)\right]+\frac{\Phi_{c}}{U} \ln \frac{H^{\prime}(s) \operatorname{sh}\left[U H^{\prime}(0)\right]}{H^{\prime}(0) \operatorname{sh}\left[U H^{\prime}(s)\right]} .
\end{aligned}
$$

Value of this force $f_{m 0}$ for initial homogeneous fluid with constant concentration of particles $\Phi_{0}$ will be equal to

$$
f_{m 0}=\Phi_{0} \int_{H^{\prime}(1)}^{H^{\prime}(0)} L\left(U H^{\prime}\right) d H^{\prime}=\frac{\Phi_{0}}{U} \ln \frac{H^{\prime}(1) \operatorname{sh}\left[U H^{\prime}(0)\right]}{H^{\prime}(0) \operatorname{sh}\left[U H^{\prime}(1)\right]} .
$$

It is convenient to describe the influence of concentration redistribution on this force by using the dimensionless coefficient $k_{m}=f_{m} / f_{m 0}$.

Some of the obtained solutions become more simple if magnetic fluid is in a magnetic saturation at large values of magnetic field intensity when $U>>1$. In this case the Langevin function $L\left(U H^{\prime}\right) \approx 1$ and the solutions of above problem take a form: $\Phi_{g}=B \exp \left(U H^{\prime}\right)$. And:

a) at the absence of phase transition

$$
B=\Phi_{0}\left[\int_{0}^{1} \exp \left(U H^{\prime}\right) d x^{\prime}\right]^{-1}, k_{m}=\frac{1}{U \Phi_{0}} \frac{\Phi_{g}(0)-\Phi_{g}(1)}{H^{\prime}(0)-H^{\prime}(1)}
$$

b) at the presence of phase transition 


$$
\begin{aligned}
& B=\Phi_{g^{*}} \exp \left[-U H^{\prime}(s)\right], \\
& \Phi_{c} s+\Phi_{g^{*}} \exp \left[-U H^{\prime}(s)\right] \int_{s}^{1} \exp \left[U H^{\prime}\left(x^{\prime}\right)\right] d x^{\prime}=\Phi_{0}, \\
& k_{m}=\frac{\Phi_{c}}{\Phi_{0}} \frac{H^{\prime}(0)-H^{\prime}(s)}{H^{\prime}(0)-H^{\prime}(1)}+\frac{1}{U \Phi_{0}} \frac{\Phi_{g}(s)-\Phi_{g}(1)}{H^{\prime}(0)-H^{\prime}(1)} .
\end{aligned}
$$

\section{Results and discussion.}

For further concrete calculations the following model approximation of distribution of magnetic field is accepted: $H^{\prime}=1 /\left(1+3 x^{\prime 2}\right)$. This approximation is based on our measurements of magnetic field intensity in a gap of real magnetic fluid seal. Compliance between this approximation and experimental data is presented in Fig.3.

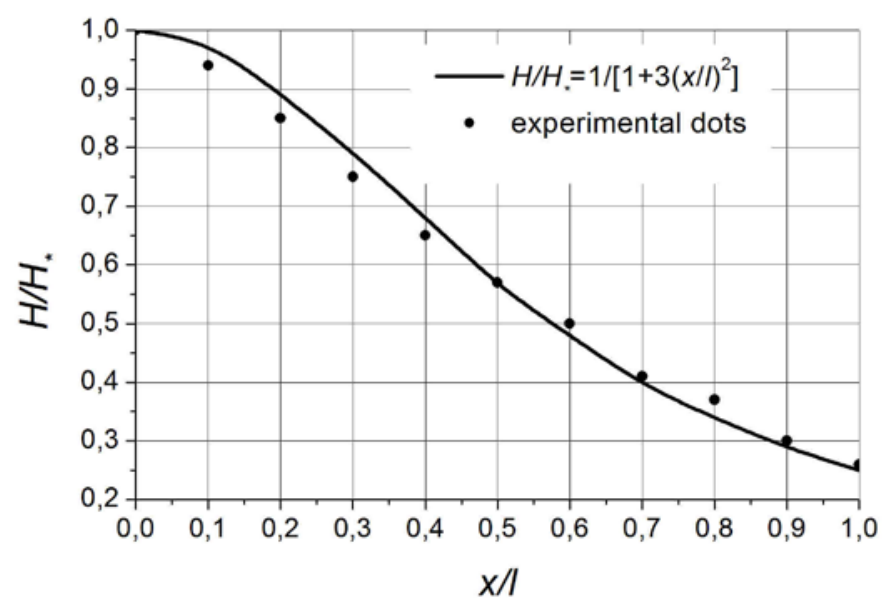

Fig. 3. Distribution of magnetic field intensity in a gap of real magnetic fluid seal. The solid line approximation by expression $H^{\prime}=1 /\left(1+3 x^{\prime 2}\right)$, dots - experimental data. $H_{*}=1,6 \cdot 10^{6} \mathrm{~A} / \mathrm{m}, l=1$ $\mathrm{mm}$.

Results of calculations fulfilled on the base of formulas (15)-(23) for $\lambda=1.5$ are presented in Figs 4-8. As it will be shown below the phase transition in magnetic fluid with reasonable average concentration of particles is observed at large values of magnetic parameter $U$, when magnetic fluid is in saturation. In this case $(\lambda=1,5$ and $L\left(U H^{\prime}\right) \approx 1$ ) the equations of the phase co-existence (10)-(11) and Fig.1 give $\Phi_{g^{*}}=0,489$ and $\Phi_{\mathrm{c}}=0,552$.

Typical distribution of particles concentration $\Phi$ in magnetic fluid depending on magnetic parameter $U$ is presented in Fig. 4 at initial average concentration $\Phi_{0}=0,1$. 


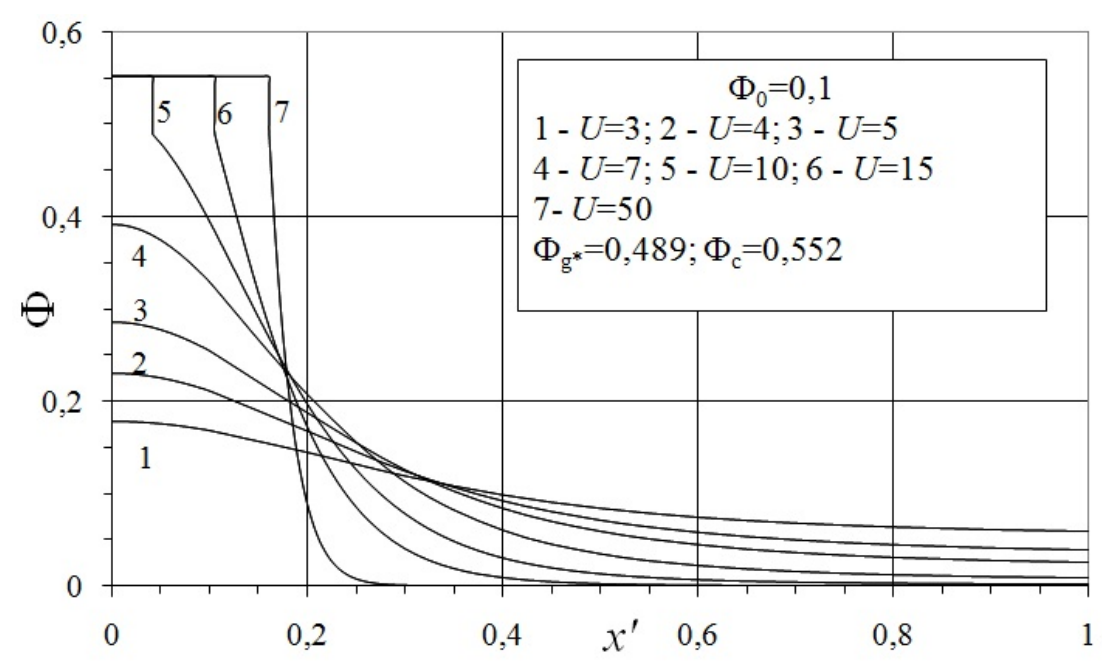

Fig. 4. Typical distribution of particle concentration $\Phi$ in magnetic fluid depending on magnetic parameter $U$.

Curves 1-4 show monotonous increasing of concentration under a magnet pole without of phase transition up to values of the magnetic parameter $U$ equal about 9 .

A large number of particles are concentrated in the region of maximum value of magnetic field intensity, and concentration of particles decreases far from it.

With increasing of $U$ concentration of particles in the region of maximum magnetic field intensity increases until reaches value $\Phi_{\mathrm{g}^{*}}=0,489$ at $U$ equal about 9 . Here the phase transition which is followed by concentration jump from $\Phi_{\mathrm{g}^{*}}=0,489$ to $\Phi_{\mathrm{c}}=0,552$ is observed.

Value $\Phi_{g^{*}}=0,489$ defining position $x^{\prime}=s$ of the phase transition interface is displaced to the right when $U$ increases. To the left of it the "condensed" phase with constant value of concentration of particles $\Phi_{c}=0,552$ takes place and the dimension of this area increases with the increasing of $U$. This fact is demonstrated by the curves 5 , 6, 7 in Fig.4.

At large values of parameter $U$, practically all number of magnetic particles is collected in area with the maximum intensity of magnetic field so the remained area becomes practically without them, that is not magnetized. In magnetic fluid seals this not magnetized volume of fluid ceases to be kept in them and can flow outside practically in the form of pure base fluid. At early stages of research of magnetic fluid seals this phenomenon was connected with not good quality of magnetic fluid. As it appears from the given results, this phenomenon can take place also in a good magnetic fluid as a result of processes of magnetophoresis, diffusion and phase transition which are of their essential attributes.

Coordinate of phase transition boundary $s$ depends also on initial average concentration of particles $\Phi_{0}$. It is obvious that with its increasing phase transition has to come at smaller values of magnetic field intensity that is at smaller values of $U$. The corresponding dependence is presented in Fig. 5. 


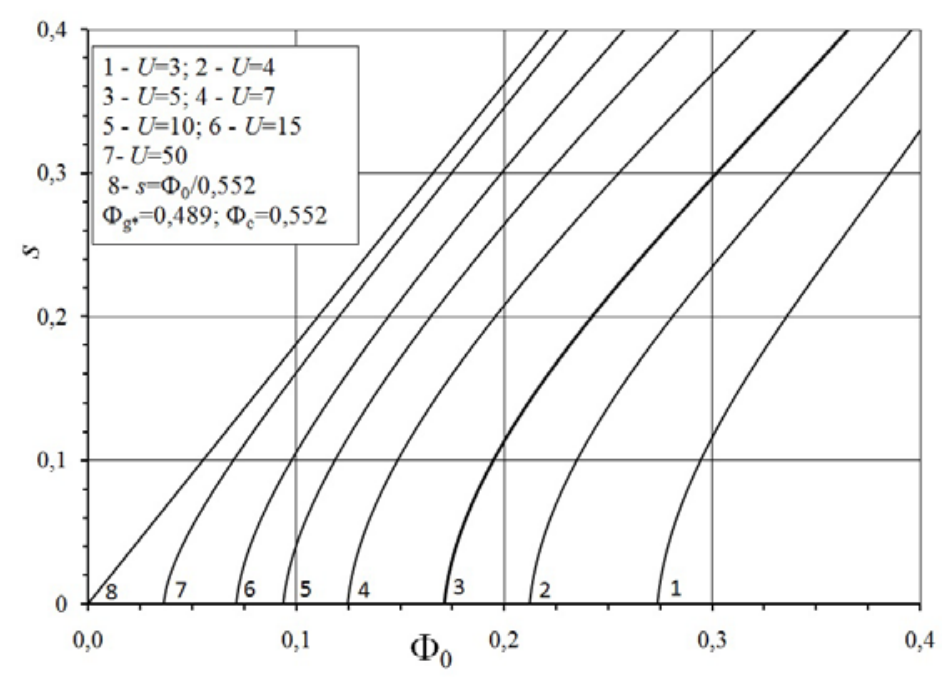

Fig. 5. Dependence of coordinate of phase transition point $s$ in magnetic fluid on average particle concentration $\Phi_{0}$ at different values of magnetic parameter $U$.

Areas to the left of the curves represented in Fig.5 correspond to the states excluding phase transition in magnetic fluid at given value of magnetic parameter $U$. Points of crossing of the curves with axis $\Phi_{0}$ define that minimum value of average concentration of particles $\Phi_{0 \min }$ at which phase transition in fluid can take place at given $U: s\left(\Phi_{0 \min }\right)=0$.

Fig.5 demonstrates that with increasing of average concentration of particles $\Phi_{0}$ at the same value of magnetic parameter $U$ the value of $s$ increases too, that is the area of the condensed phase becomes larger. The same takes place and at increasing of magnetic field intensity (magnetic parameter $U$ ) for fluid with constant value of average concentration $\Phi_{0}$. At large value of $U$ curves on the Fig. 5 tend to the straight line described by a formula (19) $s=\Phi_{0} / 0,552$, and all curves have almost the same inclination at large $s$.

The dependence of this minimum value of average concentration of particles $\Phi_{0 \min }$ on magnetic parameter $U$ is presented in Fig. 6.

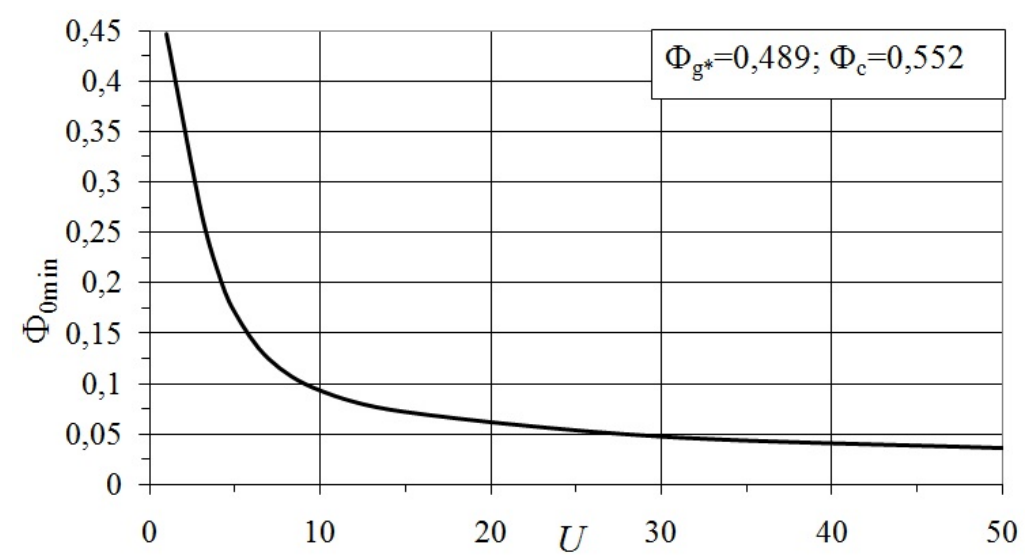

Fig. 6. Dependence of minimum value of average concentration of particles $\Phi_{0 \min }$ on magnetic parameter $U$ at which a phase transition is taken place. 
This dependence can be used also for the solution of the inverse problemdetermination of minimum value of magnetic parameter $U_{\text {min }}$ only at excess of which phase transition in magnetic fluid can take place at a given value of average concentration of particles $\Phi_{0}$.

At $U>>1$ this dependence is determined from a formula (25) as follows $\Phi_{0 \min }=\Phi_{g^{*}} \exp (-U) \int_{0}^{1} \exp \left[U H^{\prime}\left(x^{\prime}\right)\right] d x^{\prime}$.

The influence of the considered processes on the magnetic force acting on the volume of magnetic fluid, and also on force of viscous friction on moving boundary described by formulas (20) - (23), is presented in Figs. 7, 8.

As it can see from Fig.7 a strong nonlinear dependence of a dynamic coefficient of viscosity of magnetic fluid on concentration of particles described by formula of Krieger-Dougherty (13) leads to essential increase in relative value of viscous force (coefficient of viscous force $k_{\tau}$ ) with increasing of magnetic parameter $U$ when concentration of particles in the area with large intensity of magnetic field increases.

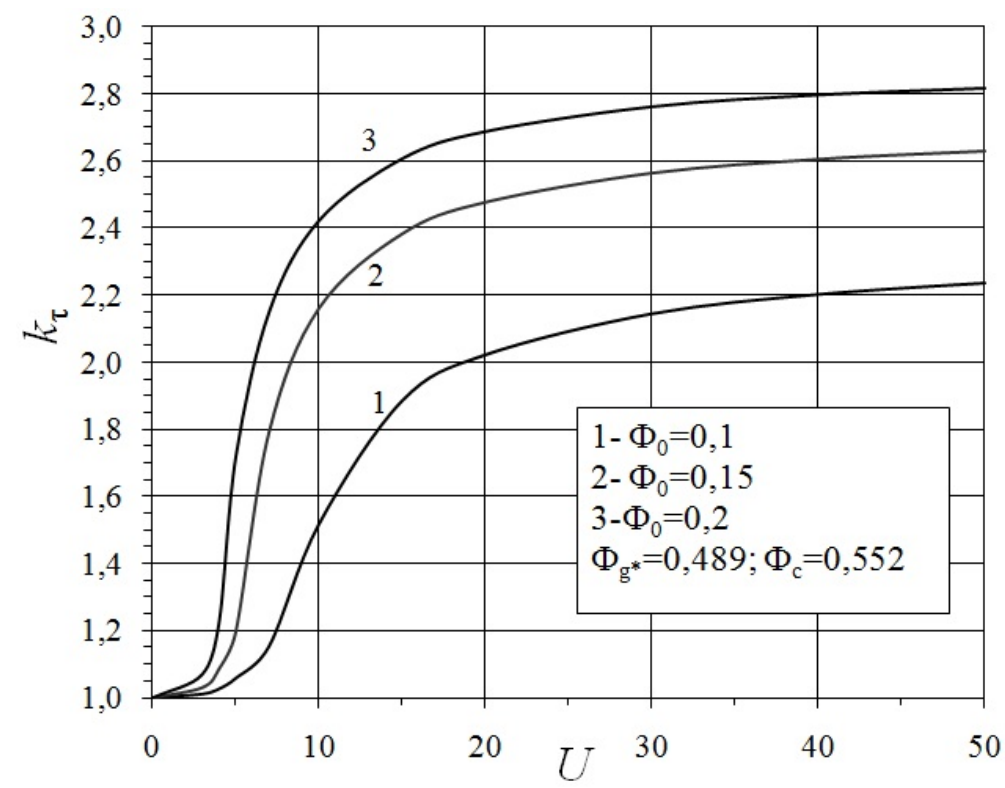

Fig. 7. Dependence of viscous force coefficient $k_{\tau}$ on magnetic parameter $U$ for different values of average concentration of particles $\Phi_{0}$.

The fast increasing of $k_{\tau}$ takes place in the range of values $U$ in which there is a phase transition in magnetic fluid and the maximum values of concentration of magnetic particles $\Phi_{g^{*}}$, and $\Phi_{c}$ further remain fixed. Further smooth growth $k_{\tau}$ is generally caused by expansion of area of the "condensed" phase of magnetic particles.

More complicated influence the redistribution of concentration of particles in magnetic fluid has on the magnetic force acting on it. It is connected with the fact that the value of this force depends not only on magnetization of fluid, but also on the value of gradient of magnetic field intensity. As a result of magnetophoresis the concentration of particles, and, therefore, magnetization of fluid, have the largest values in the point of 
maximum value of magnetic field intensity. However, as one can see from Fig.3 in this area the gradient of magnetic field intensity decreases to zero.

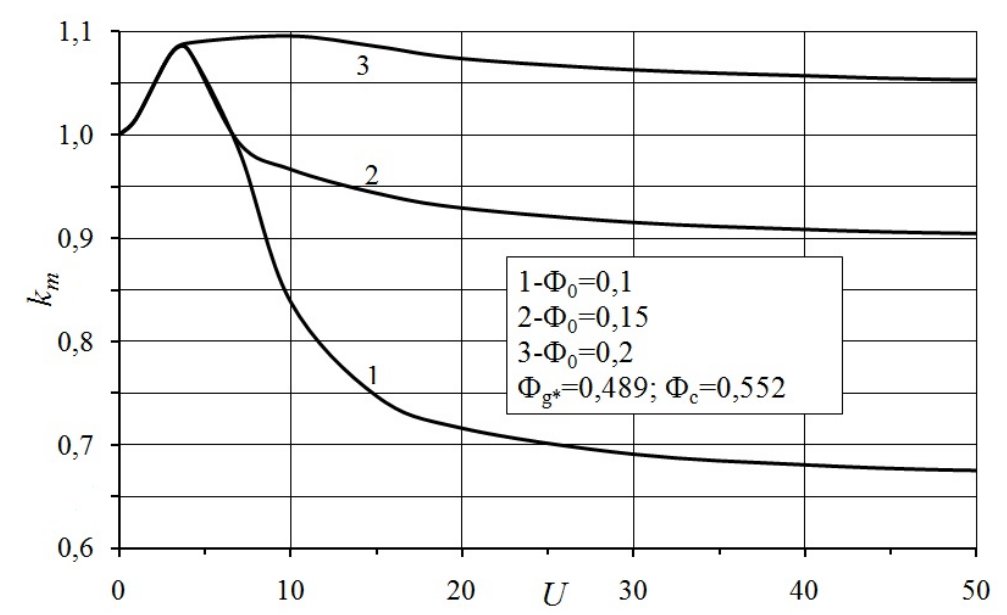

Fig. 8. Dependence of magnetic force coefficient $k_{m}$ on magnetic parameter $U$ for different values of initial average concentration of particles $\Phi_{0}$.

Therefore, as it is seen from Fig.8, at moderate values of magnetic parameter $U<$ 5 coefficient of magnetic force $k_{m}$ increases approximately by $10 \%$ due to the fact that concentration of magnetic particles in the area with large gradients of magnetic field increases. This fact was noted also earlier in [6,9]. At large values of $U$ an increasing number of particles concentrate in the area with the maximum value of magnetic field intensity, that is in the area with its minimum gradient. It leads to monotonous decreasing of coefficient of magnetic force $k_{m}$ with a further increasing of $U$. This decreasing is more visible for fluids with less average concentration of particles. In the considered situations this decreasing can reach considerable values to $40 \%$.

\section{Conclusion}

Thus, it is established that processes of magnetophoresis and Brownian diffusion can have significant effect on characteristics of flows of magnetic fluid, namely on the values of volume magnetic force and viscous tension. In this connection forces of viscous friction tend to increase, and magnetic forces can be increased, as well as decreased depending on value and configuration of the magnetic field.

Inclusion in consideration of possible phase transitions in magnetic fluid allows us to perform physically consistent calculation of characteristics of magnetic fluid flow taking into account processes of magnetophoresis and Brownian diffusion at large values of magnetic field intensity.

It is shown that phase transition in magnetic fluid is observed when the magnetic parameter exceeds a certain value, and the area of the condensed phase increases with its increasing. At large values of magnetic parameter the coordinate of phase transition interface almost linearly depends on average concentration of particles in the problem under consideration. 


\section{Acknowledgement}

The authors express their thanks to Belarusian Republican Foundation of Fundamental Researches (project No 01T17MC-013) and Russian Fund for Basic Research, 16-5812003, the Program of Russian Federation Ministry of Science and Education, project 3.1438.2017/4.6 for financial support of this work.

\section{References:}

1. R.E. Rosensweig, Ferrohydrodynamics, Cambridge University Press, New York (1985).

2. E.Blums, A.Cebers, M.M.Maiorov, Magnetic Fluids, Water de Gruyter, Berlin (1997).

3. V.G.Bashtovoy, B.M.Berkovski, A.N.Vislovich, Introduction to Thermomechanics of Magnetic Fluids, Hemisphere Publ. Corp., New York (1988).

4. S. Taketomi, Motion of ferrite particles under a high gradient magnetic field in a magnetic fluid seal, Jap. J. Appl. Phys 19 (1980) 1929-1936.

5. I.Anton, L.Vekash, I.Potents, D.Bika, Behavior of magnetic liquids in a inhomogeneous magnetic field, Magnetohydrodynamics 21 (1985) 213-217.

6. V.G. Bashtovoi, V.K. Polevikov, A.M. Algadal, The effect of diffusion processes on the statics of magnetic fluids, Proceedings of the National Academy of Sciences of Belarus 3 (2006) $42-48$.

7. V.G.Bashtovoi, V.K.Polevikov, A.E.Suprun, A.V.Stroots, S.A.Beresnev, Influence of Brownian diffusion on statics of magnetic fluid, Magnetogydrodynamics 43 (2007) 3-11.

8. V.G.Bashtovoi, V.K.Polevikov, A.E.Suprun, A.V.Stroots, S.A.Beresnev, The effect of magnetophoresis and Brownian diffusion on the levitation of bodies in a magnetic fluid, Magnetogydrodynamics 44 (2008) 121-126.

9. V.G. Bashtovoi, S.G.Pogirnitskaya, P.Kuzhir, V.M.Polunin, P.A.Ryapolov, I.A.Shabanova, A.M.Storojenko, Influence of Mass Transfer Processes on Couette Flow of Magnetic Fluid, Journal of Nano- and Electronic Physics 5 (2013) 04011.

10. W. B. Russell, D. A. Saville, W. R. Schowalter, Colloidal Dispersions, Cambridge University Press, Cambridge (1989).

11. A.Y. Zubarev, L.Y.Iskakova, Theory of structural transformations in ferrofluids: chains and gas-liquid phase transitions, Phys.Rev. E65 (2002) 061406.

12. A.Y. Zubarev, L.Y. Iskakova, Phase transitions in electro- and magnetorheological fluids, Colloid Journal 65 (2003) 159-165.

13. L.Y. Iskakova, A.Y. Zubarev, A.P. Romanchuk, On the theory of phase transitions in magnetorheological suspensions, Colloid Journal, 67 (2005) 564-572.

14. C.Magnet, P.Kuzhir, G.Bossis, A.Meunier,S.Nave, A.Zubarev, C.Lomenech, V. Bashtovoi, Behavior of nanoparticle clouds around a magnetized micro- sphere under magnetic and flow fields, Physical Review E 89 (2014) 032310. 
15. A.F. Pshenichnikov, A.S. Ivanov, Magnetophoresis of particles and aggregates in concentrated magnetic fluids, Physical Review E - Statistical, Nonlinear, and Soft Matter Physics 86 (2012) 051401.

16. A.F. Pshenichnikov, E.A. Elfimova, A.O. Ivanov, Magnetophoresis, sedimentation, and diffusion of particles in concentrated magnetic fluids, Journal of Chemical Physics, 134 (2011) 184508.

17. A.F. Pshenichnikov, A.A. Kuznetsov, Sedimentation of particles in concentrated magnetic fluids: Numerical simulation, Magnetohydrodynamics 51 (2015) 551-560.

18. N. F. Carnahan, K. E. Starling, , Equation of state for nonattracting rigid spheres, Journal of chemical physics, 51 (1969) 635.

19. K. R. Hall, Another Hard-Sphere Equation of State, J. Chem. Phys., 57 (1972) 2252.

20. Yu. A. Buyevich, A. O. Ivanov, Equilibrium properties of ferrocolloids, Physica A, 190 (1992) 276.

21. I.M. Krieger, T.J. Dougherty, Concentration dependence of the viscosity of suspensions, Trans. Soc. Rheol., 3 (1959) 137.

22. R. G. Larson, The Structure and Rheology of Complex Fluids, Oxford University Press, New York, (1999). 